Memoirs of the Coliege of Science, University of Kyoto, Series A Vol. XXVIII, Mathematics No. 3, 1954.

\title{
Note on complete local integrity domains
}

\author{
By \\ Masayoshi NAGATA
}

(Received Dec. 1, 1953)

Previously some interesting results concerning prime ideals in rings of formal power series were proved by $\mathrm{C}$. Chevalley [1]. In the present paper, we want to offer a new treatment on the similar assertions. We see on the way a new result that when $\mathfrak{v}$ is a complete (Noetherian) local integrity domain with a basic field $\boldsymbol{k}$, $\mathfrak{v}$ is separably generated ${ }^{1)}$ over $\boldsymbol{k}$ if and only if there exists a system of parameters $x_{1}, \cdots, x_{n}$ of $\mathfrak{v}$ such that $\mathfrak{v}$ is separable over the ring l. $\left\{x_{1}, \cdots, x_{n}\right\}$ (formal power series).

Throughout the present paper, a local ring means a Noetherian local ring which contains a field.

\section{$\$ 1$. Kroneckerian products.}

Let $\mathfrak{v}_{1}$ and $\mathfrak{v}_{2}$ be complete local rings with basic fields $\boldsymbol{k}_{1}$ and $\boldsymbol{k}_{2}$ respectively. If $K$ is a field containing both $\boldsymbol{k}_{1}$ and $\boldsymbol{k}_{2}$, we can define the Kroneckerian product of ( $\boldsymbol{k}_{1}$-algebra) $\mathfrak{o}_{1}$ and ( $\boldsymbol{k}_{2}$-algebra) v., over $K$, as was defined by $C$. Chevalley [2]. We denote this Kroneckerian product by $\mathfrak{o}_{1} / \boldsymbol{k}_{1} \times{ }_{K^{0}} \mathrm{o}_{2} / \boldsymbol{k}_{2}{ }^{2)}$. (For the detail, see Chevalley [2]). When $\boldsymbol{k}_{1}=\boldsymbol{k}_{2}=\boldsymbol{K}$, we denote this by $\mathfrak{o}_{1} \times{ }_{\boldsymbol{k}} \mathfrak{0}_{2}$.

We define further Kroneckerian products of complete local rings with discrete rings:

Let $\mathfrak{v}_{1}$ be a complete local ring with basic field $\boldsymbol{k}_{1}$ and let $\mathfrak{v}_{2}$ be a discrete ring ${ }^{3)}$ which contains a field $\boldsymbol{k}_{2}$. Assume that $K$ is a field which contain both $\boldsymbol{k}_{1}$ and $\boldsymbol{k}_{2}$. We define the Kroneckerian product of $\boldsymbol{k}_{1}$-algebra $\mathfrak{o}_{1}$ and discrete $\boldsymbol{k}_{2}$-algebra $\mathfrak{o}_{2}$ over $K$ as follows :

1) For the definition, see Chevalley [1] or $\$ 2$ in the present paper.

2) Though Chevalley [2] denotes this ring by $0_{1} \times_{1} 0_{0}$, we dare use a more complicated notation because the product depends on the choice of basic fields.

3) o. may be a topological ring which is not discrete; we only regard it as an abstract ring (or a discrete topological ring). 
Let $B_{1}$ be a strong base of $\mathfrak{v}_{1}$ over $\boldsymbol{k}_{1}$ and let $B^{\prime}$ be a linearly independent base of $\mathfrak{o}_{2}$ over $\boldsymbol{k}_{2}$. We set $\mathfrak{0}=\left\{\sum\left(\sum a_{\lambda n} v_{\lambda}\right) u_{n} ; a_{\lambda n} \in K\right.$, $v_{\lambda} \in B^{\prime}, u_{n} \in B_{1}, \sum a_{\lambda n} v_{\lambda}$ is a finite sum $\}$. We introduce in $v$ operations of sum and product by the followings;

$$
\begin{gathered}
\left(\Sigma\left(\sum a_{\lambda_{n}} v_{\lambda}\right) u_{n}\right)+\left(\Sigma\left(\sum b_{\lambda n} v_{\lambda}\right) u_{n}\right)=\Sigma\left(\sum\left(a_{\lambda n}+b_{\lambda n}\right) v_{\lambda}\right) u_{n}, \\
\left.\left(\Sigma\left(\sum a_{\lambda n} v_{\lambda}\right) u_{n}\right)\left(\Sigma b_{\lambda n} v_{\lambda}\right) u_{n}\right)=\Sigma\left(\sum\left(\sum a_{\lambda n} b_{\lambda^{\prime \prime}}, c_{\lambda \lambda^{\prime} x} d_{n n^{\prime} m}\right) v_{n}\right) u_{m},
\end{gathered}
$$

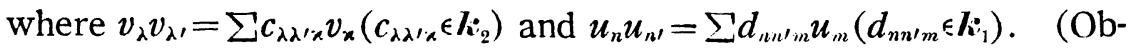
serve that $\sum a_{\lambda n} b_{\lambda^{\prime \prime}}, c_{\lambda \lambda^{\prime} x^{\prime}} d_{n n^{\prime} m}$ is a finite sum).

It is easy to. see that though this depends on the choice of strong base $B_{1}$ and linearly independent base $B^{\prime}$ of $\mathfrak{o}_{1}$ and $\mathfrak{v}_{2}$ respectively, the structure of $\mathfrak{o}$ does not depend on the choice of them. This ring $\mathfrak{o}$ is called the Kroneckerian product of (complete local) $\boldsymbol{k}_{1}$-algebra $\mathfrak{o}_{1}$ and (discrete) $\boldsymbol{k}_{2}$-algebra $\mathfrak{o}_{2}$ over $K$ and we denote this by $\mathfrak{v}_{1} / \boldsymbol{k}_{1} \times_{k}\left(\mathfrak{o}_{2} / \boldsymbol{k}_{10}\right)_{1}$. When $\boldsymbol{k}_{1}=\boldsymbol{k}_{2}=K$, we donote this by $\mathfrak{o}_{1} \times{ }_{K}\left(\mathfrak{o}_{2}\right)_{t}$. If $\mathfrak{o}_{2}=\boldsymbol{k}$ is a field, we denote this by $\mathfrak{o}_{1} \times{ }_{K} \boldsymbol{k}$.

Next we explain an easy, but, important lemma:

Lemma 1. Let $\mathfrak{v}_{1}$ and $\mathfrak{o}_{2}$ be complete local rings with the same

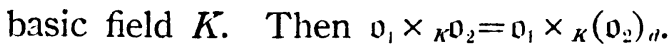

Proof. Let $B_{1}$ and $B_{2}$ be strong bases of $\mathfrak{o}_{1}$ and $\mathfrak{o}_{2}$ over $K$ respectively and let $B^{\prime}$ be a linearly independent base of $\mathfrak{o}_{2}$ over $K$. Set $\mathfrak{v}^{\prime}=\mathfrak{v}_{1} \times{ }_{K}\left(\mathfrak{v}_{2}\right)_{\mathfrak{t}}$ and $\mathfrak{v}=\mathfrak{v}_{1} \times{ }_{k} \mathfrak{v}_{2}$. Then

$$
\begin{aligned}
& \mathfrak{o}^{\prime}=\left\{\Sigma\left(\sum a_{\lambda n} v_{\lambda}\right) u_{n} ; a_{\lambda n} \in K, v_{\lambda} \in B^{\prime}, u_{n} \in B_{1}, \Sigma a_{\lambda n} v_{\lambda} \text { is a finite sum }\right\}, \\
& \mathfrak{o}=\left\{\sum b_{\mu n} w_{\mu} u_{n} ; b_{\mu n} \in K, w_{\mu} \in B_{2}, u_{n} \in B_{1}\right\} .
\end{aligned}
$$

Let $\phi$ be a mapping from $\mathfrak{v}^{\prime}$ into $\mathfrak{v}$ as follows:

$$
\phi\left(\sum\left(\sum a_{\lambda n} v_{\alpha}\right) u_{n}\right)=\sum b_{\mu n} w_{\mu} u_{n},
$$

where $\sum a_{\lambda_{n}} v_{\lambda}=\sum b_{\mu n} w_{\mu}\left(\sum b_{\mu n} w_{\mu}\right.$ may be infinite sum).

Similarly, let $\phi^{*}$ be a mapping from $\mathfrak{v}$ into $\mathfrak{v}^{\prime}$ as follows :

$$
\phi^{*}\left(\sum b_{\mu n} w_{\mu} u_{n}\right)=\sum\left(\sum a_{\lambda n} v_{\lambda}\right) u_{n},
$$

where $\sum b_{\mu_{n}} w_{\mu}=\sum a_{\lambda n} v_{\lambda}\left(\sum a_{\lambda_{n}} v_{\lambda}\right.$ must be a finite sum).

Then we see easily that $\phi$ and $\phi^{*}$ are homomorphisms and that $\phi^{\circ} \phi^{*}$ is the identity mapping. Therefore $\phi$ is an isomorphism.

\section{\$2. Separably generated extensions.}

We denote hereafter by $p$ the characteristic of the field of reference when it is not zero or the number 1 for the other case.

Definition. We say that a complete local integrity domain $\mathfrak{p}$ 
is separably generated over its basic field $\boldsymbol{k}$ if $\mathfrak{v} \times{ }_{{ }_{k}} \boldsymbol{k}^{\boldsymbol{v}^{p}}{ }^{-1}$ is an integrity domain.

Theorem 1. Let $\mathfrak{v}$ be a complete local integrity domain with a basic field k. Then the following three conditions are equivalent to each other:

(1) b is separably generated over $k$.

(2) For any strongly linearly independent subset $B$ of 1 over $\boldsymbol{k}, B_{p}=\left\{u^{p} ; u \in B\right\}$ is also strongly linearly independent over $\mathbf{k}$.

(3) For any integer $m, \mathfrak{b} \times{ }_{i k} \boldsymbol{k}^{p^{-m}}$ is an integrity domain.

Proof. Assume that $B$ is a strongly linearly independent set of $\mathfrak{v}$ over $\boldsymbol{k}$ and assume that $B^{\prime \prime}$ is not strongly linearly independent over $\mathbf{k}:$ : There exist $u_{n} \in B$ such that $\sum a_{n} u_{n}^{\nu}=0\left(u_{i} \geq u_{j}\right.$ if $i \geq j$, $\left.a_{n} \in \boldsymbol{k}\right)$, and therefore in $\mathfrak{v} \times{ }_{k} \boldsymbol{k}^{p^{-1}},\left(\sum a_{n}^{p^{-1}} u_{n}\right)^{p}=0$ and $\sum a_{n}^{p^{-1}} u_{n} \neq 0$. Therefore $\mathfrak{b} \times{ }_{l_{i}} \boldsymbol{k}^{0, p^{-1}}$ is not an integrity domain. This proves that (2) follows from (1). Next we prove the converse: Assume that $\mathfrak{v} \times{ }_{k} \boldsymbol{k}^{v^{-1}}$ contains a divisor $c$ of zero $(c \neq 0)$. Since $c^{y} \in \mathfrak{D}$, we see that $c^{p}=0$. We write $c=\sum a_{n} u_{n}\left(\left\{u_{n}\right\}\right.$ is strongly linearly independent


dent over $k$. These being settled, the eqivalence with (3) is evident.

Corollary. If a complete local integrity domain $\mathfrak{v}$ is separably generated over its basic field $\boldsymbol{k}$, , then for any integer $m, \mathfrak{b} \times{ }_{k} \boldsymbol{k}^{p^{-m}}$ is separably generated over $\mathbf{k}^{p^{-m}}$.

Remark 1. It is evident that if $\mathfrak{v}$ is a complete local 'ring with a basic field $\boldsymbol{k}$ and if $K$ is a field which contains $\boldsymbol{k}$, then $\mathfrak{b} \times{ }_{k} K$ is a complete semi-local ring; we have the identity

$$
\mathfrak{v} \times{ }_{{ }_{i}} K=\mathfrak{v} / \boldsymbol{k} \times{ }_{K} \boldsymbol{k} / \boldsymbol{l}:=\mathfrak{v} / \boldsymbol{k} \times{ }_{K} K / K \text {. }
$$

Remark 2. By Lemma 1 , we see that when $\left[\boldsymbol{k}: \boldsymbol{k}^{*^{\nu}}\right]<\infty$, a complete local integrity domain $\mathfrak{v}$ with a basic field $\boldsymbol{k}$ is separably generated over $\boldsymbol{k}:$ if and only if the quotient field of $\mathfrak{v}$ is separably generated over $k^{4} .^{4}$ In general case, we see easily that the tensor product $v \bigotimes_{l_{i}} \boldsymbol{l}^{\boldsymbol{v}^{p-1}}$ is a subring $\mathfrak{v} \times{ }_{k} \boldsymbol{k}^{\boldsymbol{p}^{p-1}}$ and therefore we see that if $\mathfrak{v}$ is separably generated over $k$, then the quotient field of $\mathfrak{v}$ is

4) We say, according to C. Chevalley [1], that a field $K$ is separably generated over its subfield $\boldsymbol{l}:$ if the tensor product $K \boldsymbol{K}_{k} \boldsymbol{h}^{p-1}$ is an integrity domain. This is equivalent to that every finitely generated extension field of $l$ contained in $K$ has a separating transcendence base over $h$ : 
separably generated over $k$. The converse is not true as is easily seen.

\section{§. Derivations.}

Definition. Let $\mathfrak{v}$ be a complete local integrity domain. A derivation $D$ of $\mathfrak{o}$ is a linear operator to the quotient field $L$ of $\mathfrak{v}$ which satisfies the following conditions:

i) $D(x y)=x D y+y D x$ (for any $x, y \in L)$,

ii) There exists an element $d(\neq 0)$ of $\mathfrak{v}$ such that $d D x \in \mathfrak{v}$ for any $x \in \mathfrak{v}$ and if $\sum u_{n}$ is a convergent series in $\downarrow$ then $\sum d D u_{n}$ is also convergent (therefore $\sum D u_{n}$ has a meaning in $\left.L\right)$ and $D\left(\sum u_{n}\right)=$ $\sum D u_{n}$.

A derivation $D$, for which it holds that $D a=0$ if $a$ is in a subring $\mathfrak{b}^{\prime}$ of $\mathfrak{b}$, is called a derivation of $\mathfrak{v}$ over $\mathfrak{v}^{\prime}$.

It is evident that the totality of derivations of $v$ (over a subring) form an $\mathfrak{v}$-module. Linear dependency of derivations is defined in this sense.

Lemma 2. Let $\mathrm{v}$ be the ring of formal power series in $x_{1}, \cdots, x_{n}$ over a field $\boldsymbol{k}$. Then the partial dervations $D_{i}=\partial / \partial x_{i}(i=1, \cdots, n)$ form a maximal linearly independent set of derivations of $\mathfrak{b}$ over $k$.

Proof is easy.

Lemma 3. Let $v$ be a complete local integrity domain with a basic field $\boldsymbol{k}$. Let $L$ be the quotient field of $\mathfrak{v}$. Assume that the characteristic $p$ of $\mathfrak{o}$ is not zero. Let $M$ be the subfield of $L$ generated by $L^{p}$ and $l:$. Take an integer $r$ such that $[L: M]=p^{r}$. Then any maximal set of linearly independent derivations of $\mathfrak{o}$ over $\boldsymbol{k}$ consists of $r$ derivations.

Proof. If $a$ is in $L^{n}, D a=0$ for any derivation $D$ of $\mathfrak{o}$. Therefore, for any derivation $D$ of $\mathfrak{b}$ over $k$ and for any element $a$ of $M$, we have $D a=0$. We take elements, $a_{1}, \cdots, a_{r}$ of $L$ such that $L=M\left(a_{1}, \cdots, a_{r}\right)$. Then we can find derivations $D_{1}, \cdots, D_{r}$ of 0 over $\boldsymbol{k}$ such that $D_{i} a_{j}=\boldsymbol{o}_{i j}$ (Kroneckerian $i$ ). That this is a maximal set of linearly independent derivations can be proved easily.

Theorem 2. Let 1 be a complete local integrity domain of dimension $n$ and with a basic field $k$. Then the number of menbers of a maximal set of linearly independent derivations of $\mathrm{o}$ over $k$ is at least $n$. It is $n$ if and only if $\mathfrak{o}$ is separably generated over $k$.

Proof. Let $x_{1}, \cdots, x_{n}$, be a system of parameters of $\mathfrak{v}$ and set $\mathrm{r}=\boldsymbol{k}\left\{x_{1}, \cdots, x_{n}\right\}$. Let $\mathfrak{v}^{\prime}$ be the totality of separably algebraic (integral) elements of $\mathfrak{v}$ over $\mathfrak{x}$. 
1) We first show that a maximal set of linearly independent derivations of $\mathfrak{v}^{\prime}$ over $\boldsymbol{k}$. consists of just $n$ numbers: It is true for. $\mathfrak{r}$ by Lemma 2 ; since $\mathfrak{v}^{\prime}$ is separable over $\mathfrak{r}$, we see that this is also true for $\mathfrak{v}^{\prime}$ (similarly to the case of the theory of fields).

2) Next we show that $\mathfrak{v}^{\prime}$ is separably generated over $\boldsymbol{k}:$ : Let $c$ be an element of $\mathfrak{v}^{\prime}$ such that $\mathrm{v}^{\prime}$ is contained in the quotient field of $\mathfrak{v}[c]$ and let $f(x)$ be the irreducible monic polynomial over $\mathfrak{o}$ satisfied by $c$. If $\mathfrak{o}^{\prime} \times{ }_{i} l_{b^{p}}{ }^{-1}$ is not an integrity domain, we see that $f(x)$ is reducible over $\boldsymbol{k}^{p^{-1}}\left\{x_{1}, \cdots, x_{n}\right\}$, which is impossible because $f(x)$ is separable and $\boldsymbol{k}^{p^{-1}}\left\{x_{1}, \cdots, x_{n}\right\}$ is purely inseparable over $\boldsymbol{k}:\left\{x_{1}, \cdots, x_{n}\right\}$.

3). We prove the general case by induction on $\left[v: v^{\prime}\right]^{\mathrm{E}}(p \geq 2)$ : Let $c_{1}, \cdots, c_{r}$ be elements of $\mathfrak{v}$ such that $c_{i}^{\prime \prime} \in \mathfrak{v}^{\prime}\left[c_{1}, \cdots, c_{i-1}\right]$, and $\left[\mathfrak{v}^{\prime}\left[c_{1}, \cdots\right.\right.$, $\left.\left.c_{s}\right]: \mathfrak{v}^{\prime}\right]=p^{x}\left(\left[\mathrm{v}: \mathfrak{v}^{\prime}\right]=p^{r}\right)$. We set $\dot{s}=\boldsymbol{k}\left\{x_{1}^{p}, \cdots, x_{n}^{p}\right\}\left[\mathrm{v}^{\prime p}\right]\left[c_{1}^{p}, \cdots, c_{r}^{\nu}\right], \mathfrak{v}^{\prime \prime}=$ $\mathfrak{v}^{\prime}\left[c_{1}, \cdots, c_{r-1}\right], \mathfrak{s}^{\prime \prime}=1:\left\{x_{1}^{p}, \cdots, x_{u}^{p}\right\}\left[\mathfrak{v}^{\prime p}\right]\left[c_{1}^{p}, \cdots, c_{r-1}^{p}\right]$. Then $\left[\because: s^{\prime \prime}\right] \leq p$, $\left[\mathfrak{v}: \mathfrak{v}^{\prime \prime}\right]=p$ and $\left[\mathfrak{v}^{\prime \prime}: \mathfrak{s}^{\prime \prime}\right] \geq p^{n}$ (by induction assumption), which shows $[0: \mathfrak{s}]=\left[0, \mathfrak{s}^{\prime \prime}\right] /\left[\mathfrak{s}: \mathfrak{s}^{\prime \prime}\right]=\left[\mathfrak{v}: \mathfrak{v}^{\prime \prime}\right]\left[\mathfrak{v}^{\prime \prime}: \mathfrak{g}^{\prime \prime}\right] /\left[\mathfrak{s}: \mathfrak{s}^{\prime \prime}\right] \geq p^{n}$. Thus we see that there exists a system of $n$ linearly independent derivations of o over $k$.

4) We assume that $\mathfrak{b}$ is separably generated oven $k$. We use the same notations as in 3). Since our assertion is true for $\mathfrak{v}^{\prime}$, we prove our assertion by induction on $\left[\mathfrak{v}: \mathfrak{v}^{\prime}\right]$. Since $\mathfrak{v}^{\prime \prime}$ is a subspace of $\mathfrak{v}$, we see that $\mathfrak{v}^{\prime \prime}$ is also separably generated. Therefore by our induction assumption we see that $\left[0^{\prime \prime}: s^{\prime \prime}\right]=p^{n}$. Therefore we have only to show that $\left[\mathfrak{s}: \mathfrak{s}^{\prime \prime}\right]=p$. Let $B$ be a strong base of $\mathfrak{v}^{\prime \prime}$ over $\boldsymbol{k}$. Then $\left\{B, B c_{r}, \cdots, B c_{r}^{p-1}\right\}$ form a strong base of $\mathfrak{v}^{\prime \prime}[c]$ over $k$. If $\left[\mathfrak{s}_{:}: \mathfrak{s}^{\prime \prime}\right]=1$, we must have a relation $\sum_{i=0}^{p-1} b_{i} c_{r}^{p i}=0\left(b_{i} \in \mathfrak{s}^{\prime \prime}, b_{0} \neq 0\right)$. Then we must have a relation $\sum a_{\lambda i} u_{\lambda}^{\prime} \lambda_{r}^{p_{i}}=0\left(a_{\lambda i} \in k_{i}, u_{\lambda} \in B, 0 \leq i \leq p-1\right.$, $\left.\sum a_{\lambda i} u_{\lambda}^{\nu}=b_{i}\right)$, which is a contradiction to our assumption that $\mathfrak{v}$ is separably generated over $\boldsymbol{l}$ : (because $\mathfrak{b}^{\prime \prime}[c]$ is a subspace of $\mathfrak{o}$ ).

5) Conversely, we assume that a maximal set of linearly independent derivations over $k$ consists of just $n$ members. We can take a set of linearly independent derivations $D_{1}, \cdots, D_{n}$ over $\boldsymbol{k}$ and a system of elements $c_{1}, \cdots, c_{n}$ of $\mathfrak{b}$ such that $D_{b} c_{j}=i_{i j}$ (Kroneckerian i). We may assume that these $c_{i}$ are unit in $\mathrm{v}$, because if $c_{i}$ is not a unit, we may take $1+c_{i}$ instead of $c_{i}$. It is evident that $x_{1}^{p}, \cdots, x_{n}^{p}$ is a system of parameters of $\mathfrak{o}$. We set $y_{i}=c_{i} x_{i}^{p}$.

5) $\left[0: \mathfrak{o}^{\prime}\right]$ means the index of the quotient field of $\mathfrak{v}$ over that of $\mathfrak{o}^{\prime}$, 
Then $y_{1}, \cdots, y_{n}$ form a system of parameters of $\mathfrak{v}$. Then by our construction, we see easily that every derivations of $\boldsymbol{k}\left\{y_{1}, \cdots, y_{n}\right\}$ over $\boldsymbol{k}$ can be uniquely extended to a derivation of $\mathfrak{p}$ over $\boldsymbol{k}$. This shows that $\mathfrak{v}$ is separable over $\boldsymbol{k}\left\{y_{1}, \cdots, y_{n}\right\}$. Now we see that $\mathfrak{v}$ is separably generated over $k$ by virtue of 2) above.

We have proved in the same time (in 2) and 5)) the following

Theorem 3. Let o be a complete local integrity domain with a basic field $\mathbf{k}$. Then $\mathrm{o}$ is separably generated over $\boldsymbol{k}$ if and only if there exists a system of parameters $x_{1}, \cdots, x_{n}$ of o such that $\mathrm{v}$ is separable over $k\left\{x_{1}, \cdots, x_{n}\right\}$.

Now we prove

Theorem 4. Assume that a complete local integrity domain 0 is separably generably generated over its basic field $k$. If $K$ is an extension field of $k$ such that $\boldsymbol{k}$ is separably algebraically closed in $K$, then $\mathfrak{0} \times{ }_{k} K$ is an integrity domain.

Proof. By virtue of Theorem 3, we can choose a system of parameters $x_{1}, \cdots, x_{n}$ of $\mathfrak{n}$ so that $\mathfrak{v}$ is separable over $\boldsymbol{k}\left\{x_{1}, \cdots, x_{n}\right\}$. We choose an element $c$ of $\mathfrak{v}$ so that $\left[\mathfrak{v}: \mathfrak{k}\left\{x_{1}, \cdots, x_{n}\right\}[c]\right]=1$ and let $f(x)$ be the irreducible monic polynomial over $\boldsymbol{k}\left\{x_{1}, \cdots, x_{n}\right\}$ satisfied by $c$. If $\mathfrak{v} \times{ }_{l i} K$ is not an integrity domain, we have that $f(x)$ is reducible over $K\left\{x_{1} ; \cdots, x_{n}\right\}: f(x)=g(x) h(x)$, where $g$ and $h$ are monic polynomiais over $K\left\{x_{1}, \cdots, x_{n}\right\}$. Then every coefficients of $g$ and $h$ are integral over $k\left\{x_{1}, \cdots, x_{n}\right\}$, therefore they are in $\boldsymbol{k}^{\boldsymbol{w}^{-m}}\left\{x_{1}, \cdots, x_{n}\right\}$ for some integer $m$, which shows that $0 \times{ }_{k} \boldsymbol{k}^{p^{-m}}$ is not an integrity domain and this is a contradiction to that 0 is separably generated over $\boldsymbol{k}$.

\section{$\$ 4$. Regular extensions.}

Definition. A complete local integrity domain o with a basic field $\boldsymbol{k}$ is said to be a regular extension of $\boldsymbol{k}$ if 1 ) $\boldsymbol{k}$ is algebraically closed in the quotient field of $\mathfrak{v}$ and 2) $\mathfrak{v}$ is separably generated over $l$.

Theorem 5. Let o be a complete local integrity domain with a basic field k. Then the following three conditions are equivalent to each other:

(1) $\mathrm{o}$ is a regular extension of $k$.

(2) $\mathfrak{o}$ is separably generated over $k$ and for any finite separable extension $k k^{\prime}$ of $k, \mathfrak{v} \times l_{l} k^{\prime}$ is an integrity domain.

(3) For any finite separable extension $\boldsymbol{k}^{\prime \prime}$ of $\boldsymbol{k}^{p-1}, \mathfrak{v} \times{ }_{k} \boldsymbol{l}^{\prime \prime}$ is an integrity domain. 
Proof is easy.

Remark. We see easily that if a complete local integrity domain $\mathfrak{v}$ with a basic field $\boldsymbol{k}$ is a regular extension of $\boldsymbol{k}$, then the quotient feld of $\mathfrak{v}$ is regular extension of $\boldsymbol{k}^{(i)}$ The converse is true if $\left[k: k^{\prime \prime}\right]<\infty$. (See the remark at the end of $\$ 2$.)

Theorem 6. Let $\mathfrak{v}$ be a complete local integrity domain with a basic field $\boldsymbol{k}$. Assume that $\mathrm{o}$ is a regular extension of $\mathrm{k} .{ }^{6}$. Then for any field $K$ containing $k, v \times{ }_{i} K$ is an integrity domain. Further i) ${ }_{1} K$ is a regular extension of $K$.

Proof. That $0 \times{ }_{k i} K$ is an integrity domain can be proved by a similar way as in the proof of Theorem 4 . Let $K^{\prime}$ be an arbitrary field containing $K$. Then $\left(\mathfrak{v} \times{ }_{i k} K\right) \times{ }_{K} K^{\prime}=\mathfrak{v} \times{ }_{i k} K$ is an integrity domain, which shows that $0 \times{ }_{k} K$ is a regular extension of $K$.

\section{$\S 5$. An application.}

Theorem 7. Let $\mathfrak{v}_{1}$ and $\mathfrak{v}_{2}$ be complete local integrity domains with the same basic field $\boldsymbol{k}$. Assume that $\mathfrak{D}_{1}$ is a regular extension of $\boldsymbol{k}$. Then $\mathfrak{v}_{1} \times{ }_{1 i} \mathrm{D}_{2}$ is an integrity domain. In this case, if $\mathfrak{b}_{2}$ is also a regular extension of $k$, then $D_{1} \times{ }_{k} D_{2}$ is a regular extension of $k$.

Proof. Let $L$ be the quotient field of $\mathfrak{0}_{2}$. Then $\mathfrak{0}_{1} \times{ }_{k} L$ is an integrity domain. By Lemma $1,0_{1} \times{ }_{k i} b_{2}$ is a subring of $\mathfrak{v} \times{ }_{k i} L$, which shows that $\mathfrak{o}_{1} \times{ }_{1,0}$, : is an integrity domain. Now we assurne that $\mathfrak{o}_{2}$ is also a regular extension of $k$. Let $K$ be an arbitrary field containing $k$. Then $\left(\mathfrak{b}_{1} \times{ }_{k} \mathfrak{v}_{2}\right) \times{ }_{k} K=\left(\mathfrak{v}_{1} \times{ }_{k} K\right) \times{ }_{K}\left(\mathfrak{o}_{2} \times{ }_{k} K\right)$. Since $\mathfrak{v}_{1} \times{ }_{k} K$ is a regular extension of $K$ and since $v_{2} \times{ }_{k} K$ is an integrity domain, we see that $\left(\mathfrak{v}_{1} \times{ }_{1} \mathrm{v}_{2}\right) \times{ }_{k} K$ is an integrity domain. This shows that $v_{1} \times k_{i},:$ is a regular extension of $\boldsymbol{k}$.

Corollary 1 . Let $\mathfrak{v}_{1}$ and $\mathfrak{v}_{2}$ be complete local integrity domains with basic fields $\boldsymbol{k}_{1}$ and $\boldsymbol{k}$. respectively. Assume that $K$ is a field containing both $\boldsymbol{k}_{1}$ and $\boldsymbol{k}_{\mathrm{g}}$. Then $\mathfrak{n}_{1} / \boldsymbol{k}_{1} \times{ }_{{ }_{1}} \boldsymbol{v}_{1} / \boldsymbol{k}_{2}$ is a regular extension of $K$ if $\mathfrak{v}_{1}$ and $v_{1}$ are regular extensions of $\nu_{1}$ and $\delta_{2}$ respectively.

Corollary 2. Let $x_{1}, \cdots, x_{n}, y_{1}, \cdots, y_{m}$ be analytically independent eiements over a field $\boldsymbol{k}$ and let $p_{1}$ and $p_{2}$ be prime ideals of $\mathfrak{v}_{1}=\boldsymbol{k}\left\{x_{1}, \cdots, x_{n}\right\}$ and $\mathfrak{v}_{2}=\boldsymbol{k}\left\{y_{1}, \cdots, y_{m}\right\}$ respectively. Then $\left(\mathfrak{p}_{1}, \mathfrak{p}_{2}\right) \boldsymbol{k}\left\{\boldsymbol{x}_{1}, \cdots x_{n}, y_{1}, \cdots, \boldsymbol{y}_{m}\right\}$ is prime if $\mathfrak{o}_{1} / \mathfrak{p}_{1}$ is a regular extension

6) We say that a field $K$ is a regular extension of its subfield $k$ : if the tensor produst $K \chi_{i} k$ of $K$ and the algebraic closure $\bar{k}$ of $k$ over $\boldsymbol{k}$ is an integrity domain, or equivalently, if $K$ is separably generated over $\boldsymbol{k}$ : and if $\boldsymbol{k}$ : is algebraically closed in $K$. 
of $\boldsymbol{k}$. If furthermore, $v_{2} / \downarrow_{2}$ is also a regular extension of $\boldsymbol{k}$, then $\boldsymbol{k}\left\{x_{1}, \cdots, x_{n}, y_{1}, \cdots, y_{m}\right\} /\left(p_{1}, p_{2}\right) \boldsymbol{k}\left\{x_{1}, \cdots, x_{n}, y_{1}, \cdots y_{n}\right\}$ is a regular extension of $\boldsymbol{k}$.

\section{BIBLIOGRAPHY}

1. C. Chevalley, Some properties of ideals in rings of power series, Trans. Amer. Math. Soc. 55 (1944), pp. 68-84.

2. C. Chevalley, Intersections of algebraic and algebroid varities, Trans. Amer. Math. Soc. 57 (1945), pp. 1-85. 